Journal of Animal and Veterinary Advances 9 (14): 1913-1917, 2010

ISSN: $1680-5593$

(C) Medwell Journals, 2010

\title{
The Morphology of the Os Penis in the Adult Mouse
}

\author{
${ }^{1}$ Dincer Yildiz, ${ }^{2}$ Durmus Bolat and ${ }^{1}$ Siyami Karahan \\ ${ }^{1}$ Department of Anatomy, Faculty of Veterinary Medicine, \\ Kirikkale University, Campus, Yahsihan, Kirikkale, Turkey \\ ${ }^{2}$ Department of Anatomy, Faculty of Veterinary Medicine, Selcuk University, Selcuklu, Konya
}

\begin{abstract}
This study focused on morphology of the os penis in adult mouse (Mus musculus domesticus). The os penis was located within the corpus cavernosum penis and extended up to the half way of the glans penis. With a close resemblance to that of the rat os penis, the shape of the mouse os penis appeared a probe-like structure. Histologically, the body of os penis was consisted of compact bone and a narrow bone marrow. Hyaline cartilage constituted the proximal end, to which the corpus cavernosum penis attached and blended with the perichondrium. A completely ossified proximal end was rarely observed. The organization of hyaline cartilage at the proximal end resembled the growth plate of the long bone. However, chondrocytes did were not well organized into columns as in the growth plate. Like in the physis of the long bone, invasion of hyperthropic chondrocyte by blood vessels originating from the underlying bone was present; however, chondrocytes embedded directly in osteoid matrix were also quite common. While the proximal end of the os penis was covered with hyaline cartilage, the distal bony end was continuous with a type of tissue varied from loose to dense connective tissue and to fibrocartilage-like tissue. At the distal bony end, newly synthesized osteoid matrix was distinguishable. Thus, the os penis in the adult mouse is a dynamic structure exhibiting continuous growth both at the proximal and distal ends.
\end{abstract}

Key words: Morphology, os penis, adult, mouse, osteoid matrix, Konya

\section{INTRODUCTION}

Os penis is a bony structure that is situated within the Corpus cavernosum penis (Barone, 1990; Getty, 1975; Gultiken et al., 2004; Evans and Christensen, 1993; Dinc et al., 1996; Atalar and Ceribasi, 2006).

Many carnivores, insectivores, rodents, certain bats, seals, porcupine and a few primates have os penis of different length and shape (Barone, 1990; Baryshnikov et al., 2003; Gultiken et al., 2004; Hebel and Stromberg, 1986; Pamukoglu and Cakir, 2001; Rasmussen et al., 1986). In an adult rat, for example, it is 5.5-7.0 $\mathrm{mm}$ in length (Murakami, 1987).

The ureathral groove that runs along the longitudinal axis of the ventral aspect of the os penis is of clinical importance especially in the dog (Gultiken et al., 2004; Rasmussen et al., 1986).

The os penis can be divided into a proximal and a distal part (Getty, 1975; Gultiken et al., 2004). In the rat, the proximal part of the os penis was made of hyaline cartilage (Dinc et al., 1996) to which fibers originating from the Corpus cavernosum penis tightly adhere
(Vilmann, 1982). While ossification in the proximal part of the os penis in the rat occurs as membranous structure by day 1 post-partum, cartilage structure increases in the proximal part approximately by day 8 and cartilage structure almost nearly turns to bony structure by day 100 (Vilmann, 1982).

Cartilage process of the os penis in the rat is located in the distal part of the penile blastema and consisted of groups of cell which are closed to each other and are slightly distinguished from surrounding tissues.

Getty (1975) reported that the distal end of the os penis is consisted of hyaline cartilage in the young rats while adults had fibrous cartilage; Rasmussen et al., 1986; Vilmann and Vilmann, 1983; Yilmaz et al., 1993).

Information on the mouse os penis is very limited. As the mouse is a frequently used specie in biomedical studies, detailed information on the mouse os penis may bear importance for comparative medicine and studies on bone formation and remodeling. Thus, the aim of the study was to depict the gross and histological features of the mouse os penis in detail.

Corresponding Author: Dincer Yildiz, Department of Anatomy, Faculty of Veterinary Medicine, Kirikkale University, Campus, Yahsihan, Kirikkale, Turkey 


\section{MATERIALS AND METHODS}

In this study, 20 adults mice (Mus musculus domesticus) aged 100-130 days were sacrificed by sodium pentobarbitone and preserved in $10 \%$ formol solution. The study was approved by the ethic committee of Kirikkale University. The os penis was separated from the radix penis and carefully dissected out. Upon gross examination, the os penis was processed for histology. In gross examination, the length of the os penis was measured by a digital compass (Kyoto) and stereomicroscope (Olympus SZ 6045) with an ocular micrometer. Macroscopic samples were photographed by Fuji Fine Pix 602 digital photograph machine. For histology, tissue samples were decalcified in 3\% nitric acid solution.

After washing in running tab water, samples were dehyradated through graded alcohol series and embedded in paraffin. Tissue samples in paraffin blocks were cut serially at a thickness of $6 \mu \mathrm{m}$ on a rotary microtome. Once stained with hematoxylin and eosin or Crossmann's triple stain, sections were examined under an Olympus $5 \times 50$ light microscope; Statistical analyses of samples were performed by using Minitab ${ }^{\varpi}$ release version 12.1. Statistical analyses were done by using two sample t-test.

\section{RESULTS AND DISCUSSION}

Macroscopic results: The mouse penis was typically constituted of corpus cavernosum penis, corpus cavernosum glandis and urethra and it was enclosed by the preputium (Fig. 1 and 2). The penis made a prominent

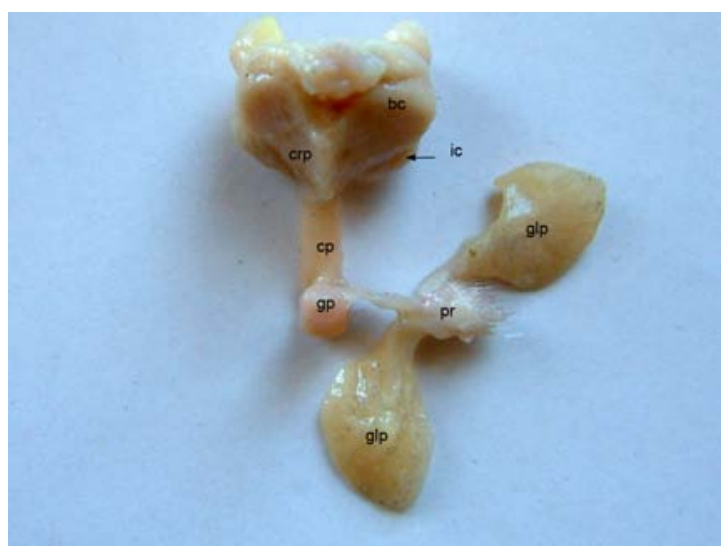

Fig. 1: The mouse os penis and associated structures: $\mathrm{bc}=$ bulbocavernosus muscle; ic $=$ ischiocavernosus muscle; $\mathrm{crp}=$ crus penis; $\mathrm{cp}=$ corpus penis; $\mathrm{gp}=$ glans penis; $\mathrm{glp}=$ preputial gland; $\mathrm{pr}=$ preputium flexura at some point when the corpus penis joined to the glans penis. The corpus penis projects cranioventrally, however the glans penis bended caudoventrally with a prominent flexura. The length of the penis, the distance between the beginning of corpus penis and the tip of the glans penis, was $9.82 \pm 1.06 \mathrm{~mm}$ ( $\mathrm{SE} \pm$ Mean $0.24 \mathrm{~mm}$ ).

The glans penis constituted only a small part (approximately $3 \mathrm{~mm}$ ) of this length. The os penis began after the flexura (Fig. 2) and extended up to the half way of the penis length.

The os penis was continuous with a dense connective tissue structure. The length of the os penis, excluding the dense connective tissue at the distal part, was $1.693 \pm 0.033 \mathrm{~mm}$ (SE \pm Mean $0.034 \mathrm{~mm}$ ). At the bottom surface, the ureathral groove run along the length of the os penis (Fig. 3). The depth of the urethral groove did change at the entire length.

Microscopic results: The corpus cavernosum penis was easily distinguished from the surrounding structures by its cavern feature (Fig. 4). Below the os penis, urethra was observed and its epithelial lining varied from stratified squamous epithelia to pseudo stratified squamous epithelia. The venous sinuses were located around the os penis and filled by erythrocytes (Fig. 4 and 5).

The corpus cavernosum penis was attached to and continuous with the os penis (Fig. 4). The loose connective tissue fibers originating from the corpus cavernosum penis merged with the periochondrium of hyaline cartilage located at the proximal end of the os penis (Fig. 4). The perichondrium covering the proximal end of the os penis merged with the periosteum, the thin connective tissue layer that wraps the os penis (Fig. 4). The cells of the outer layer of the perichondrium were relatively flat while the cells of the inner layer were more

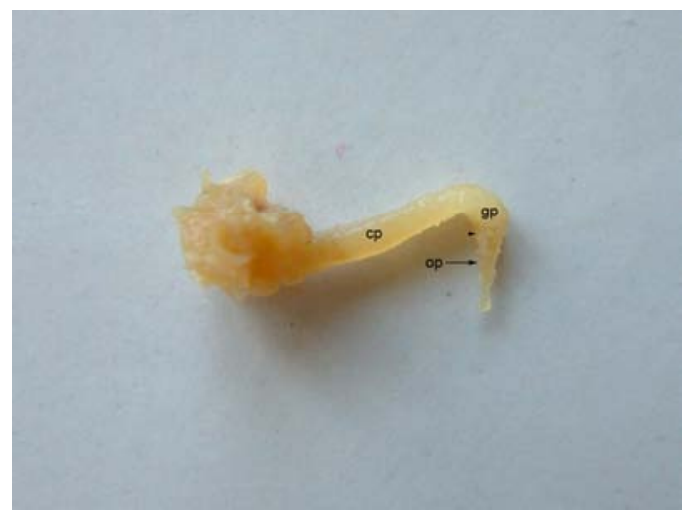

Fig. 2: The mouse os penis, lateral view. $\mathrm{cp}=$ corpus penis, $\mathrm{gp}=$ glans penis; $\mathrm{op}=\mathrm{os}$ penis 


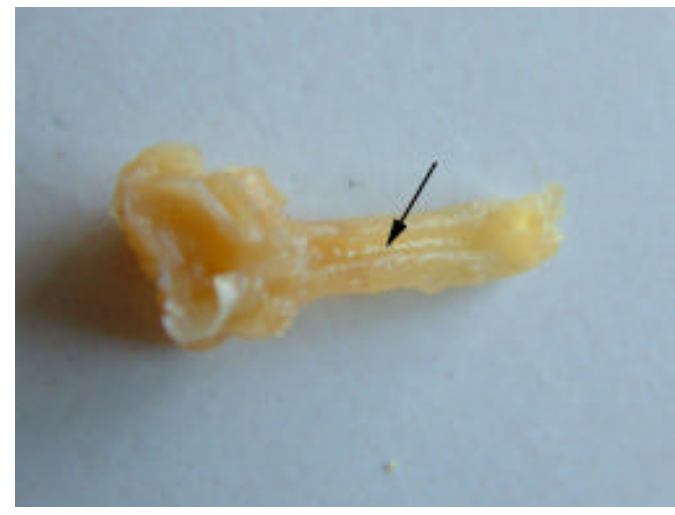

Fig. 3: The mouse os penis, ventral view. The arrow indicates the urethral grove

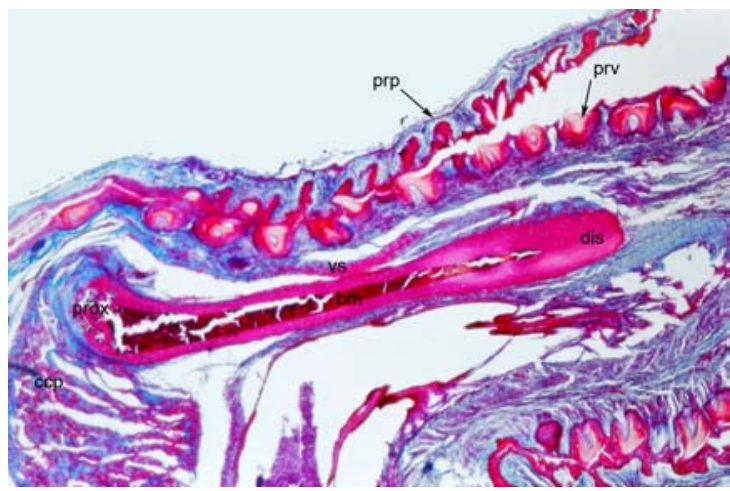

Fig. 4: A histological view of saggitally sectioned os penis. $\mathrm{ccp}=$ corpus cavernosum penis; prox $=$ proximal end; dis = distal end; vs = venous sinus; $\operatorname{prp}=$ parietal layer of preputium; prv $=$ visceral layer of the preputium. Crossmann triple staining; bar $=360 \mu \mathrm{m}$

rounded (Fig. 6). The chondrocytes located adjacent to the bony osteoid were more hyperthropic. In some instances, hyperthropic chondrocytes were invaded by blood vessels originating from the underlying bone was observed. Chondrocytes embedded in osteoid matrix near the hyaline matrix were also common (Fig. 6).

Rarely did chondrocytes arrange in columns as in the growth plate (physis) of long bones (Fig. 7). Except at the proximal end of the os penis at which bone was mainly of spongious type, the os penis was composed of lamellar bone (Fig. 5). Cortical lamella and a few haversian channels were observed. The bone had bone marrow and smooth endosteal surface (Fig. 5). At the distal end, newly synthesized osteoid matrix was quite distinguishable in some instances (Fig. 8). The tissue adjacent to the distal bony end varied from loose to dense connective tissue, hyaline cartilage-like tIssue to fibro cartilage with

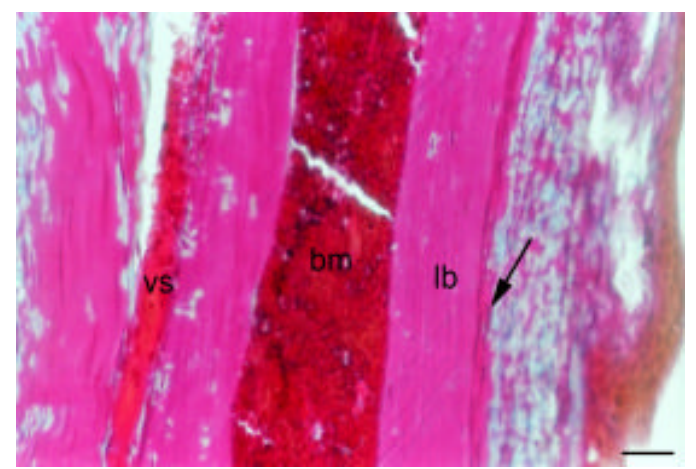

Fig. 5: Histologic view of the body of the os penis. $b m=$ bone marrow; $\mathrm{vs}=$ venous sinus; $\mathrm{lb}=$ lamellar bone. Crossmann triple staining; bar $=150 \mu \mathrm{m}$

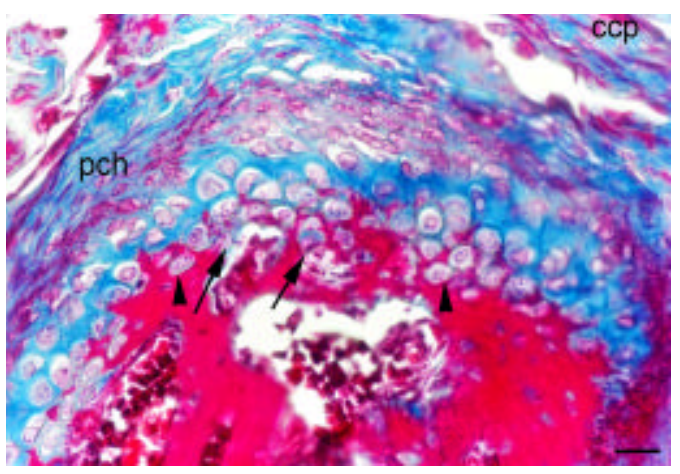

Fig. 6: A histologic view of the proximal end of the os penis. Corpus cavernosum penis (ccp) continous with perichondrium (pch). The hyperthropic chpndrocytes located near the underlying bone is either being invaded (arrows) or embedded directly in osteoid matrix (arrow heads). Crossmann triple staining; bar $=70 \mu \mathrm{m}$

numerous chondrocytes (Fig. 8 and 9). Cells embedded in relatively newly synthesized matrix at the tip of the distal bony end had variable morphology, flat to round. At the distal bony end, a clearly discernable single layer of osteoblasts was not observed.

The mouse penis is completely resembles the rat penis in morphologic features. It is characteristic for the rodentia that glandula preputialis are present and corpus penis turns cranio-ventrally and then turns to caudoventrally making a flexura and ends with the glans penis. As it observed in the present study, like that of the rat the mouse os penis is a probe-like structure and extends down to the half way of the glans penis length. At the distal end, the os penis bends ventrally. Many carnivores, insectivores, rodents, certain bats, seals, porcupine and 


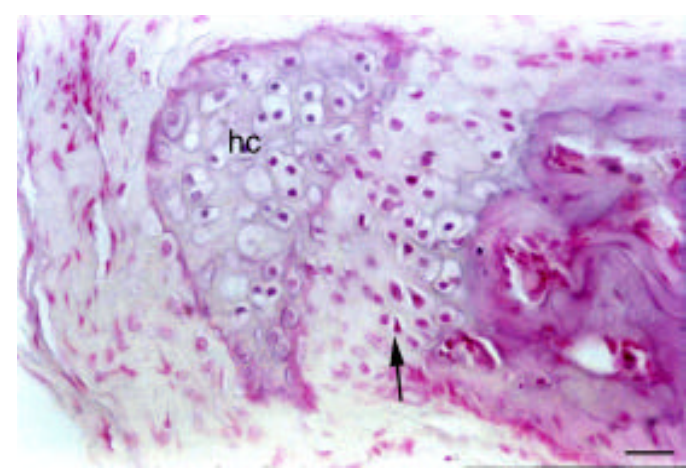

Fig. 7: Hyperthropic chondrocytes in the proximal end of the os penis. Chondrocytes throughout the entire hyalin cartilage (hc) are hyperthropic; thus, the proximal end is in the processes of complete ossification. Hematoxylin and Eosin bar $=140 \mu \mathrm{m}$

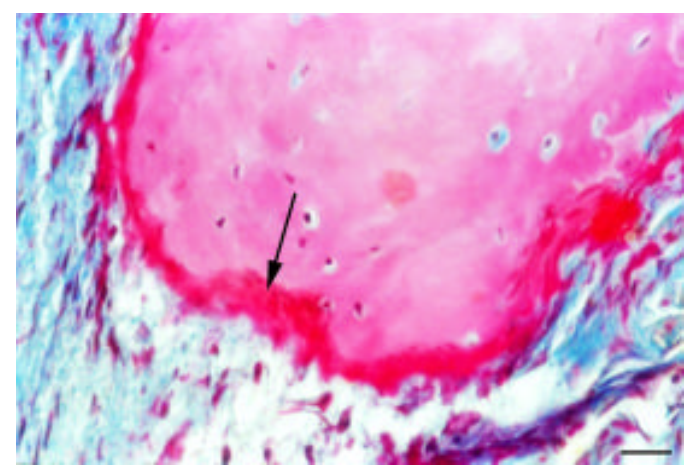

Fig. 8: A histologic view of the distal end of the os penis. The newly synthesized osteoid matrix (arrow) at the distal end can be distinguished by different staining intensity. Crossmann triple staining; bar $=70 \mu \mathrm{m}$

a few primates have os penis of different length and shape (Barone, 1990; Baryshnikov, 2003; Gultiken et al., 2004; Hebel and Stromberg, 1986; Pamukoglu and Cakir, 2001; Rasmussen et al., 1986). For example, in the adult rat it is 5.5-7.0 $\mathrm{mm}$ in length (Murakami, 1987). In the adult mouse, the length of os penis is considerably smaller, approximately $1.7 \mathrm{~mm}$.

As described by Yilmaz et al. (1993), the os penis could be bipartite or even trifurcate in rodents and have synovial joints. However, we were not able observe such structures in the mouse os penis. The urethral groove is of clinical importance especially in the dog as it prevents the compression on the urethra (Gultiken et al., 2004; Rasmussen et al., 1986). Bony growths on the surface of the urethral groove interfere with flow of urine and ejaculate and are of clinical importance because of its

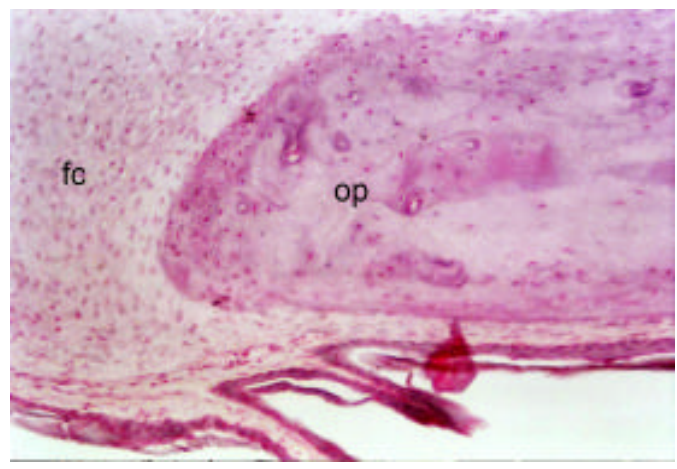

Fig. 9: The distal end of the os penis. The tissue located in front of the os penis varied from loose to dens connective tissue to fibrocartilage (fc). Hematoxylin and Eosin bar $=140 \mu \mathrm{m}$

obstruction of calculi passing through the urethra (Evans and Christensen, 1993). While the urethral groove in the dog looses its depth and widens towards the distal end, it is relatively wide open in the red fox along its entire length (Dinc et al., 1996; Gultiken et al., 2004). In the mouse, however, the urethral groove does not exhibit a significant change in the depth along the entire length.

Although, variable types of connective tissues occupy the proximal end of the os penis during the development and growth process, it is hyaline cartilage in nature in the adult rat and become ossified approximately at the 100th day of life (Yilmaz et al., 1993). The mice included in the present study were 100-130 days old. We observed hyaline cartilage at the proximal end of the os penis although, a complete ossification was observed in a few number of mice. Thus, the growth of the mouse os penis at the proximal end may continue up to the 130th day of age. The elements of the endochondrial bone formation can be seen at the proximal end of the os penis. The hyaline cartilage with hypethrophic chondrocytes adjacent to the underlying bone closely resembles the physis of the long bone. Invasion of hyperthropic chondrocytes by blood vessels originating from the underlying bone is quite common but chondrocytes embedded in the osteoid matrix are also frequent. Unlike in the physis of the long bone, in which chondrocytes in the resting zone proliferate, the perichondrium in the os penis covers the proximal end and it seems the origin of chondrocytes in hyaline cartilage.

The perichondrium at the top of the os penis is continuous with the periosteum, a layer of tissue with progenitor cells that can differentiate to osteoblasts. At the distal end, relatively newly synthesized osteoid matrix that can be differentiated by the difference in staining intensity can be observed. Thus, the os penis is still a 
dynamic structure with a continuous growth at both ends and in width in adult mice aged 130 days. By this continuous growth, the mouse os penis is similar to the rodent long bones. As described by Karahan (2002), the growth plate in the long bones of the rodents remains unossified in adulthood.

In contrast to the proximal end of the os penis which is of hyaline cartilage, the tissue located cranial to the distal end of the os penis had a variable morphology: a loose type of connective tissue to dense, hyaline cartilage-like tissue with numerous cells to fibrocartilage. The question is what determines the presence of such tissue structures in adult mice.

Another important point regarding the distal end of the os penis is that what the origin of cells in osteoid matrix is. In the present study, the cells within or near the osteoid matrix does not express a complete osteoblastic morphology but varied from spindle to round in shape although, a few cells may have an osteoblastic appearance.

The question is how such cells can change morphology and survive when they are completely embedded in mineralized bone matrix. Yamamato (1989) raised a similar question in the rat. Endochondrial ossification in fibrocartilage of the distal segment of the os penis was introduced by Izumi et al. (2000).

\section{CONCLUSION}

In this study, the mouse os penis a dynamic structure with a continuous growth at the proximal and distal ends. The proximal end is composed of hyaline cartilage while the tissue attaching the distal bony end varied from loose to dense, hyaline-like to fibrocartilage.

\section{REFERENCES}

Atalar, O. and A.O. Ceribasi, 2006. The morphology of the penis in porcupine. Veterinari Medicina, 51: 66-70.

Barone, R., 1990. Anatomie Comparee Des Mammifieres Domestiques. VIGOT, Paris, pp: 247-259.

Baryshnikov, G.F., O.R.P. Bininda-Emond and A.V. Abramov, 2003. Morphological variability and evokution of the baculum(os penis) in mustelidae (carnivora). J. Mammol., 84: 673-690.
Dinc, G., S. Yilmaz, A. Girgin and A. Aydin, 1996. Light microscopic studies on the os penis of the rat. Firat Univ J. Health Sci., 10: 149-152.

Evans, E.H. and C.G. Christensen, 1993. Miller's Anatomy of the Dog. 3nd Edn., W.B. Saunders, Philadelphia, pp: $517-524$.

Getty, R., 1975. The Anatomy of Domestic Animals. 5th Edn., W.B. Saunders Co., Philadelphia, pp: 1583.

Gültiken, M.E., D. Yildiz and D. Bolat, 2004. The anatomy of the red fox (Vulpes vulpes). Vet. J. Ankara Univ., 51: 71-73.

Hebel, R. and M.W. Stromberg, 1986. Anatomy and Embriology of the Laboratory Rat. Biomedical Verlag, Federal Rebuplic of Germany, pp: 78-79.

Izumi, K., I. Yamaoka and R. Murakami, 2000. Ultrasucture of the developing fibracartilagea of the os penis of rat. J. Morphol., 243: 187-191.

Karahan, S., 2002. Development of in vivo models of osteoarthritis and evaluation of exogenous glycosaminoglycans and exercise on osteoarthritic rat stifle joint, bone density and expression of neuropeptides and receptors. Ph.D. Thesis, Auburn University, AL.

Murakami, R., 1987. A histological study of the development of the penis of wild-type and androgen insensitive mice. J Anat., 153: 223-231.

Pamukoglu, N. and A. Cakir, 2001. Os penis (baculum) in badger (Meles meles). Vet. J. Ankara Univ., 48: $139-140$.

Rasmussen, K.K., H. Vilmann and M. Juhl, 1986. Os penis of the rat $\mathrm{V}$. the distal cartilage process. Acta Anat., 125: 208-212.

Vilmann, A. and H. Vilmann, 1983. Os penis of the rat IV. The proximal growth cartilage. Acta Anat, 117: $136-144$

Vilmann, H., 1982. Os penis of the rat III. formation and growth of the bone. Acta Morphol. Neerl Scand., 20: 309-318.

Yilmaz, O., H. Yildiz, A. Bahadir, B. Yildiz and A. Serbest, 1993. The comparative study of os penis (Baculum) of Turkish and German shepered dog. Uludag Univ. J. Fac. Vet. Med., 12: 62-67.

Yamamato, M., 1989. An electron microscope study of the distal segment of the os penis of the rat. Arch. Histol. Cytol., 52: 529-541. 